Rômulo Cristovão de Souza'

Rejane Sobrino Pinheiro"

Cláudia Medina Coeli"I

Kenneth Rochel de Camargo

Jr."'

Tânia Z Guillén de Torres"
Curso de Graduação em Enfermagem. Fundação Educacional Serra dos Órgãos. Teresópolis, RJ, Brasil

" Departamento de Medicina Preventiva. Faculdade de Medicina. Universidade Federal do Rio de Janeiro. Rio de Janeiro, RJ, Brasil

III Instituto de Medicina Social. Universidade Estadual do Rio de Janeiro. Rio de Janeiro, RJ, Brasil

Correspondência | Correspondence: Rômulo Cristovão de Souza Curso de Graduação em Enfermagem Fundação Educacional Serra dos Órgãos Av. Alberto Torres, 111 - Alto 25964-004 Teresópolis, RJ, Brasil

E-mail: romulocsouza@oi.com.br

\section{Aplicação de medidas de ajuste de risco para a mortalidade após fratura proximal de fêmur}

\author{
Risk adjustment measures for \\ mortality after hip fracture
}

\section{RESUMO}

OBJETIVO: Analisar os fatores associados à mortalidade em pacientes para tratamento da fratura proximal do fêmur e as diferentes formas de ajuste de risco.

MÉTODOS: Estudo de coorte não-concorrente com 390 pacientes com 50 anos ou mais. Os pacientes foram identificados no Sistema de Informações Hospitalares do Sistema Único de Saúde, internados para correção cirúrgica da fratura proximal do fêmur em um hospital universitário do município do Rio de Janeiro, entre 1995 e 2000. Foram levantados dados complementares nos prontuários desses pacientes e elaborados modelos de regressão logística para a chance de óbito em 90 dias após a data da admissão, segundo perfil dos pacientes e do tratamento. Foram calculados índices de classificação da gravidade do paciente.

RESULTADOS: A taxa de mortalidade foi de 7,4\% e os fatores que tiveram efeito sobre o óbito foram: idade (OR=1,06; IC 95\%: 1,02;1,11), quantidade de comorbidades $(\mathrm{OR}=1,44$; IC 95\%: 1,12;1,69), índice de comorbidade de Charlson acima de $2(\mathrm{OR}=6,67$; IC 95\%: 2,98;22,16) e intervalo de tempo para a realização da cirurgia $(\mathrm{OR}=1,04$; IC 95\%:1,02;1,07).

CONCLUSÕES: A quantidade de comorbidades e o índice de comorbidade de Charlson auxiliaram na predição da mortalidade.

DESCRITORES: Fraturas do fêmur, epidemiologia. Fraturas do fêmur, mortalidade. Risco ajustado. Fatores de risco. Estudos de coortes. 


\begin{abstract}
OBJECTIVE: To assess factors associated to mortality in patients with hip fracture and to describe different risk adjustment measures.

METHODS: Non-concurrent cohort study comprising 390 patients aged 50 years and more. Patients were identified from the Brazilian Unified Health System Hospital Information System, admitted for hip fracture surgery in a teaching hospital in Rio de Janeiro, southeastern Brazil, between 1995 and 2000. Data from medical records were collected and analyzed by logistic regression models to study 90-day mortality odds after admission according to patient and treatment profiles. Severity of illness classification indexes were estimated.
\end{abstract}

RESULTS: Mortality rate was $7.4 \%$ and factors affecting mortality were age $(\mathrm{OR}=1.06 ; 95 \% \mathrm{CI}: 1.02 ; 1.11)$, number of co-morbidities $(\mathrm{OR}=1.44 ; 95 \%$ CI: 1.12;1.69), Charlson co-morbidity index (OR=6.67; 95\% CI: 2.98;22.16) and time to surgery $(\mathrm{OR}=1.04 ; 95 \% \mathrm{CI}: 1.02 ; 1.07)$.

CONCLUSIONS: Number of co-morbidities and Charlson co-morbidity index helped predicting the mortality rate. KEY WORDS: Femoral fractures, epidemiology. Femoral fractures,
mortality. Risk adjustment. Risk factors. Cohort studies.

\section{INTRODUÇÃO}

A fratura proximal de fêmur (FPF) é uma importante causa de morbidade e mortalidade entre a população idosa. ${ }^{6,11,17,22}$ Nos anos de 1997 e 1998, foram internados 66 mil pacientes na Inglaterra e País de Gales por esse motivo. ${ }^{4}$ Nos Estados Unidos, são registradas mais de 350 mil fraturas de fêmur anualmente, representando um custo aproximado de seis bilhões de dólares. ${ }^{13} \mathrm{Com}$ o envelhecimento da população, esses valores tendem a crescer, podendo dobrar até o ano de $2040 .{ }^{25}$

A taxa de mortalidade associada à FPF em idosos é alta, variando entre $12 \%$ e $37 \%$ em um ano após a fratura. ${ }^{16,25} \mathrm{O}$ risco de morte é maior entre o segundo e sexto meses depois da fratura, período no qual a taxa média de mortalidade é de aproximadamente $11 \%{ }^{16}$ Uma revisão conduzida por Sakaki et $\mathrm{al}^{22}$ apresenta taxas de mortalidade para diferentes intervalos de tempo após a FPF: 5,5\% para a mortalidade hospitalar, 4,7\% em até 30 dias após a fratura, 11,9\% em três meses, $19,2 \%$ em um ano e $24,9 \%$ em dois anos.

Pessoas com mais idade têm mais chance de morrer após FPF. As taxas de óbito esperadas são maiores entre os homens, apesar da FPF ser mais freqüente em mulheres. ${ }^{11,16,25}$

Diversos estudos apresentam a capacidade cognitiva como fator de predição da mortalidade após fratura

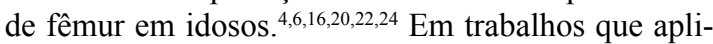
caram testes de avaliação do estado mental, pacientes com melhores resultados sobreviveram mais após uma fratura proximal de fêmur. ${ }^{6,16}$
Não existe consenso sobre a influência do tempo na mortalidade para a realização da cirurgia após a fratura. Enquanto alguns autores encontraram aumento na chance de óbito em função de um maior intervalo de tempo para a realização da cirurgia, outros trabalhos que analisaram esse tempo de espera não comprovaram a sua relação com uma maior mortalidade..$^{2,12,16,22}$ Outros fatores não mostraram associação com a mortalidade após FPF, como o tipo de procedimento cirúrgico e o tipo de anestesia utilizado. ${ }^{22}$ A respeito do tipo de anestesia, O'hara et al, ${ }^{19}$ depois de analisarem dados de 9.425 pacientes, também não encontraram associação entre a anestesia e morbimortalidade.

Diferenças encontradas nas taxas de mortalidade entre hospitais podem ocorrer em função do perfil de gravidade da população atendida, devendo ser aplicada uma estratégia para controlar e ajustar este indicador por variáveis que afetem o resultado. Poucos discordam da necessidade de se considerar um ajuste de risco de óbito, baseado na classificação da gravidade do paciente, mesmo não havendo uma definição de qual a melhor maneira de fazê-lo. ${ }^{14}$ Diversos sistemas de classificação de gravidade têm sido propostos, variando em função dos métodos de classificação dos pacientes, definição de gravidade e dos dados necessários para o seu uso. ${ }^{14}$

O Índice de Comorbidade de Charlson (ICC) é um método de classificação de gravidade que utiliza dados dos diagnósticos secundários para atribuir um risco de morte ao paciente. Esse método foi desenvolvido a 
partir de uma coorte de 604 pacientes em um hospital nos Estados Unidos para predizer a mortalidade em um ano. O ICC foi validado em uma coorte de 685 mulheres com câncer de mama, acompanhadas por dez anos, sendo difundido e empregado em diversos trabalhos. ${ }^{3,7}$ Esse método consiste de uma lista de 19 condições clínicas anotadas como diagnóstico secundário, com o objetivo de medir a gravidade do caso e avaliar o seu efeito sobre o prognóstico do paciente. Para cada uma dessas condições, é atribuída uma pontuação, representada por um peso que varia entre um e seis. ${ }^{3}$ Para gerar um escore único, a idade do paciente também tem um peso: a partir dos 50 anos, cada período de dez anos corresponde a um ponto adicional no índice.

Outro índice que tem sido utilizado em alguns trabalhos para classificar o paciente, segundo a sua gravidade, é o da American Society of Anesthesiology (ASA). Esse índice possui uma escala de classificação que varia de um a cinco, representando o risco cirúrgico, baseado no estado físico do paciente. É um sistema amplamente aceito de classificar o paciente de acordo com o seu risco pré-operatório e utilizado como preditor de resultados, embora não seja considerado um método de ajuste de risco. ${ }^{10}$

A classificação ASA aparece freqüentemente em estudos sobre a mortalidade após FPF, ${ }^{11,16,20,23}$ que mostraram que essa chance de óbito aumenta entre os pacientes com maiores escores. Conforme Stoddart et al, ${ }^{23}$ a taxa de mortalidade em 90 dias após a FPF é de 5,3\% para pacientes classificados como ASA 1 ou 2 , e $22,4 \%$ para pacientes classificados como ASA 3 ou 4. Os achados de Gdalevich et a ${ }^{11}$ indicam sobrevivência menor em pacientes com escores ASA acima de 2.

As doenças coexistentes (comorbidades) têm sido utilizadas como preditoras da mortalidade após FPF., ${ }^{416} \mathrm{O}$ efeito das comorbidades sobre a mortalidade tem sido medido tanto pela quantidade de doenças coexistentes, quanto pelo seu tipo. Pacientes com maior número de doenças coexistentes têm maior possibilidade de morrer. ${ }^{6,24}$ As doenças cardíacas, pulmonares, renais, acidente vascular cerebral e diabetes mellitus são as mais fortemente relacionadas com o desfecho. ${ }^{22}$

O objetivo do presente trabalho foi analisar os fatores associados à mortalidade de pacientes internados em até 90 dias após a fratura proximal de fêmur e as estratégias de ajuste de risco.

\section{MÉTODOS}

Foi realizado um estudo de coorte não concorrente no período de 1995 a 2000, com pacientes de 50 anos ou mais, internados em um hospital universitário (HU), no município do Rio de Janeiro. O HU tem capacidade operacional instalada de 527 leitos, dos quais 243 são destinados à clínica cirúrgica. Foram selecionadas nas bases de dados do Sistema de Informações Hospitalares do Sistema Único de Saúde (SIH-SUS) todas as internações cirúrgicas para correção da FPF (código 820 da Classificação Internacional de Doenças, nona revisão). No caso de mais de uma fratura no período de análise, apenas a primeira internação foi considerada. Utilizou-se formulário padronizado para captação nos prontuários de informações sociodemográficas dos pacientes, características da fratura, tipo de cirurgia, tipo de anestesia, índice ASA, comorbidades e complicações cirúrgicas. Foram excluídos da análise casos de fratura patológica ou cujo diagnóstico de fratura proximal de fêmur não era confirmado após revisão do prontuário. Foram incluídos 390 registros na amostra final.

A base de hospitalizações foi relacionada com as bases de dados de mortalidade obtidas no Departamento de Dados Vitais da Secretaria de Estado de Saúde do Rio de Janeiro. O relacionamento foi realizado pelo método probabilístico, por meio do programa RecLink II. ${ }^{5} \mathrm{O}$ ICC foi calculado segundo a adaptação de D'Hoore et al, ${ }^{7}$ empregando um programa para essa finalidade.*

Foram construídos três modelos de regressão logística para a mortalidade em 90 dias, cada um deles considerando uma das variáveis para o ajuste de risco: ICC, Índice ASA e quantidade de comorbidades. Não foi possível elaborar um modelo único com as três variáveis, devido à forte correlação entre elas, criando instabilidade no processo de modelagem. As variáveis idade e quantidade de comorbidades foram tratadas como contínuas, o ICC foi agrupado em 0 a 1, 2 e 3 ou mais, e o índice ASA foi categorizado como 1 a 2 e 3 ou mais. Essa categorização para ASA foi feita por haver poucas observações para a classificação 4 .

Foram incluídas no modelo completo as variáveis que apresentaram significância estatística $(\alpha<0,20)$. A eliminação das variáveis desse modelo se deu para as de menor significância estatística, considerando $\alpha=0,05$. A retirada de uma variável foi feita após a comparação das deviances dos modelos com e sem a variável em questão.

Para fins de análise dos dados, foram utilizados os pacotes estatísticos Stata 8.0 e SPSS 10.0.

O projeto foi aprovado pelo Comitê de Ética em Pesquisa do Instituto de Estudos de Saúde Coletiva, Universidade Federal do Rio de Janeiro.

\section{RESULTADOS}

Dos 390 pacientes estudados (Tabela 1), 286 (70,8\%) eram do sexo feminino e $114(29,2 \%)$ do masculino. A idade média encontrada foi de 74,4 anos, com desvio-

* Camargo Jr KR, Coeli CM. CalcCharlson, versão 1.1 [software na Internet]. Rio de Janeiro: Universidade do Estado do Rio de Janeiro.

Disponível em http://paginas.terra.com.br/educacao/kencamargo/RecLink.html [Acesso em 21 maio 2007] 
Tabela 1. Características dos pacientes internados em um hospital universitário por fratura proximal de fêmur. Rio de Janeiro, RJ, 1995-2000. N=390

\begin{tabular}{|c|c|c|}
\hline Variável & $\mathrm{N}$ & $\%$ \\
\hline \multicolumn{3}{|l|}{ Sexo } \\
\hline Masculino & 114 & 29,2 \\
\hline Feminino & 276 & 70,8 \\
\hline \multicolumn{3}{|l|}{ Idade (anos) } \\
\hline 50 a 59 & 38 & 9,8 \\
\hline 60 a 69 & 82 & 21,0 \\
\hline 70 a 79 & 116 & 29,7 \\
\hline 80 a 89 & 131 & 33,6 \\
\hline 90 a 99 & 23 & 5,9 \\
\hline \multicolumn{3}{|c|}{ Média 74,8 (desvio-padrão 10,7) } \\
\hline \multicolumn{3}{|l|}{ Tipo de fratura } \\
\hline Colo de fêmur & 149 & 38,2 \\
\hline Intertrocantérica & 209 & 53,6 \\
\hline Subtrocantérica & 30 & 7,7 \\
\hline Outras & 2 & 0,5 \\
\hline \multicolumn{3}{|l|}{ Tipo de cirurgia } \\
\hline Fixação interna & 252 & 64,6 \\
\hline Colocação de prótese & 135 & 34,6 \\
\hline Sem informação & 3 & 0,8 \\
\hline \multicolumn{3}{|l|}{ Tipo de anestesia } \\
\hline Geral & 55 & 14,2 \\
\hline Localizada & 283 & 72,5 \\
\hline Geral + local & 45 & 11,5 \\
\hline Sem informação & 7 & 1,8 \\
\hline \multicolumn{3}{|l|}{ Índice ASA } \\
\hline 1 & 24 & 6,2 \\
\hline 2 & 202 & 51,8 \\
\hline 3 e mais & 135 & 34,6 \\
\hline Sem informação & 29 & 7,4 \\
\hline \multicolumn{3}{|l|}{$\mathrm{N}^{\circ}$ de comorbidades } \\
\hline 0 & 67 & 17,2 \\
\hline 1 & 103 & 26,4 \\
\hline 2 & 105 & 26,9 \\
\hline 3 & 57 & 14,6 \\
\hline 4 & 40 & 10,3 \\
\hline 5 & 11 & 2,8 \\
\hline 6 & 2 & 0,5 \\
\hline 7 & 3 & 0,8 \\
\hline 9 & 2 & 0,5 \\
\hline \multicolumn{3}{|c|}{ Índice de Comorbidade de Charlson } \\
\hline 0 e 1 & 290 & 74,4 \\
\hline 2 & 71 & 18,2 \\
\hline 3 e mais & 29 & 7,4 \\
\hline Total & 390 & 100,0 \\
\hline
\end{tabular}

ASA: American Society of Anesthesiology padrão de 10,6 anos. O tipo de fratura mais comum foi a intertrocantérica $(53,6 \%)$, seguida pela de colo de fêmur $(38,2 \%)$, subtrocantérica $(7,7 \%)$ e outros tipos $(0,5 \%)$. A fixação interna foi o procedimento cirúrgico mais utilizado $(64,6 \%)$ e depois a colocação de prótese $(34,6 \%)$. O tipo de anestesia mais freqüente foi o geral $(72,5 \%)$. Mais da metade dos casos $(51,8 \%)$ foi classificada, segundo o índice ASA, como 2. O escore de ICC mais alto encontrado foi o $4(2,1 \%)$. Em torno de $70 \%$ dos pacientes apresentaram até duas comorbidades e apenas $4,6 \%$ deles tinham cinco ou mais doenças coexistentes. A quantidade média de comorbidades encontrada foi de 1,9. Aplicando o ICC, $74,4 \%$ dos pacientes receberam uma pontuação entre 0 e $1,18,2 \%$ a pontuação 2 e apenas $7,4 \%$ foram classificados com um escore 3 ou mais. $O$ tempo médio de permanência foi de 20,5 dias, com um valor mediano de 17 dias. O maior tempo de internação encontrado foi de 170 dias. $\mathrm{O}$ tempo médio entre a fratura e a admissão foi de 2,9 dias (mediana de 2 dias), o tempo médio de espera para a cirurgia desde a admissão foi de 12,7 dias (mediana 11 dias) e o tempo médio de espera para a cirurgia desde a fratura foi de 14,8 dias (mediana de 13 dias).

No período estudado, 99 pacientes $(25,4 \%)$ morreram e a taxa de mortalidade em noventa dias foi de $7,4 \%$ (29 óbitos). A Tabela 2 apresenta os três modelos de regressão para associação com mortalidade. O modelo de associação do ICC com a mortalidade, mostrou que as variáveis com efeito sobre o desfecho foram a idade (OR=1,06; IC 95\%: 1,02;1,11), o tempo de espera para a cirurgia (OR=1,04; IC 95\%:1,02;1,07) e ICC acima de 2 (OR=6,67; IC 95\%:2,98;22,16). Assim, cada ano de vida a mais do paciente representou um aumento na chance de morrer em torno de $6 \%$. O acréscimo de um dia no tempo de espera para a realização da cirurgia aumentou a chance óbito em cerca de $4 \%$. Essa chance foi maior, também, para escores ICC mais altos, chegando a ser 6,6 vezes maior quando esse escore foi superior a dois, em relação ao grupo com ICC zero ou um.

Pelo segundo modelo que avaliou o efeito da quantidade de comorbidades, observou-se efeito semelhante para as variáveis idade e tempo entre a fratura e a cirurgia. A coexistência de uma doença a mais representou elevação na chance de óbito de aproximadamente $44 \%$ $(\mathrm{OR}=1,44$; IC 95\%: 1,12;1,69).

O terceiro modelo, utilizado para analisar o efeito do índice ASA na mortalidade em 90 dias, mostrou associação entre a mortalidade e a idade e o tempo até a cirurgia, semelhantes às encontradas nos dois outros modelos. As chances de óbito em 90 dias para pacientes com ASA 3 ou 4 não foram estatisticamente significativas (OR=1,71; IC 95\%:0,76;3,88). 
Tabela 2. Modelos de regressão logística para ajuste de risco de mortalidade em 90 dias após fratura proximal de fêmur. Rio de Janeiro, RJ, 1995-2000.

\begin{tabular}{|c|c|c|c|}
\hline Modelo & Coeficiente $(\beta)$ & Odds ratio & IC 95\% \\
\hline \multicolumn{4}{|c|}{ Índice de Comorbidade de Charlson } \\
\hline Idade & 0,06 & 1,06 & 1,$02 ; 1,11$ \\
\hline Tempo para cirurgia & 0,04 & 1,04 & 1,$02 ; 1,07$ \\
\hline \multicolumn{4}{|l|}{ Charlson } \\
\hline 2 & 0,28 & 1,34 & 0,$57 ; 4,49$ \\
\hline 3 e mais & 1,84 & 6,27 & 2,$98 ; 22,16$ \\
\hline \multicolumn{4}{|l|}{ Comorbidades } \\
\hline Idade & 0,05 & 1,05 & 1,$01 ; 1,10$ \\
\hline Tempo para cirurgia & 0,04 & 1,04 & 1,$02 ; 1,07$ \\
\hline Quantidade & 0,36 & 1,44 & 1,$12 ; 1,69$ \\
\hline \multicolumn{4}{|l|}{ Índice ASA } \\
\hline Idade & 0,05 & 1,05 & 1,$00 ; 1,10$ \\
\hline Tempo para cirurgia & 0,04 & 1,04 & 1,$01 ; 1,07$ \\
\hline ASA 3 e 4 & 0,54 & 1,71 & 0,$76 ; 3,88$ \\
\hline
\end{tabular}

Categorias de referência: ASA 1 e 2; Charlson 0 e 1.

\section{DISCUSSÃO}

A mortalidade em 90 dias após a FPF encontrada no presente trabalho foi de $7,4 \%$, próximo ao relatado por Nather et $\mathrm{al}^{17}(6,4 \%)$ e por Cree et $\mathrm{ll}^{6}(8 \%)$, porém mais baixo do que o de Stoddart et $\mathrm{al}^{23}(10 \%)$ e de Sakaki et $\mathrm{al}^{22}(11,9 \%)$.

A idade, o sexo, as comorbidades, o índice ASA e a deficiência cognitiva são citados como os fatores mais fortemente associados à mortalidade após FPF., 4, 17,20,22,23 Embora o tempo de espera para a realização da cirurgia tenha sido discutido por alguns autores, ${ }^{2,12,18,23,25}$ não há consenso se esse tempo tem efeito sobre o risco de morte.

Os resultados confirmam a associação da idade com a mortalidade após FPF, indicando que a chance de morrer foi maior em pacientes mais idosos, o que também pode ser visto em outros estudos. ${ }^{4,6,8,18,24}$

O sexo é apontado por diversos autores como uma variável associada com a mortalidade após FPF, com maiores taxas esperadas de óbito entre homens. Quando compararam grupos de pessoas que sofreram FPF com outras sem fratura, Fransen et $\mathrm{al}^{9}$ acharam uma chance de óbito 1,5 vez maior no grupo das mulheres e sete vezes maior entre os homens. No presente trabalho, o sexo não teve efeito estatisticamente significante sobre a chance de morrer embora tenha havido mais de mortes entres as mulheres. Resultados similares foram encontrados por van Balen et al, ${ }^{24}$ estudando 102 pacientes na Holanda, que não associaram o sexo com a mortalidade; e Nurmi et al, ${ }^{18}$ que encontraram mortalidade maior entre as mulheres $(34 \%$ contra
$28 \%$ ), porém, essa diferença não foi estatisticamente significativa. As hipóteses para o maior percentual de óbito entre os homens seriam a maior possibilidade de queda e maior número de comorbidades. Mas, esse último não se aplica no presente trabalho, por ter sido controlado na modelagem.

Um importante fator de predição da mortalidade encontrado no presente estudo foi o tempo de espera para a cirurgia. Essa associação tem sido apontada pela literatura, 2,12,18,23,25 contudo, não existe um consenso sobre o papel do tempo de espera para ser operado sobre a chance de óbito. Grimes et $\mathrm{al}^{12}$ analisaram dados de 8.383 pacientes com fratura de fêmur nos Estados Unidos entre 1983 e 1993, dividindo-os em três grupos: operados em 24 horas após a FPF, e operados após 24 horas sem ou com patologia ativa. Os pacientes com patologia ativa levaram mais tempo para serem operados por estarem clinicamente instáveis pelas doenças. A mortalidade, inicialmente, foi maior entre aqueles operados com menos de 24 horas, entretanto, essa diferença desapareceu após o ajuste pela presença de doenças coexistentes. Casaletto \& Gatt ${ }^{2}$ compararam pacientes operados em até um dia e após um dia da admissão e observaram que a mortalidade em um ano foi mais elevada para aqueles cujo tempo de espera para a cirurgia foi maior, principalmente em pessoas acima de 80 anos de idade.

O tempo de espera entre a fratura e a operação foi em média de 14,8 dias, com uma mediana de 13 dias, tempos superiores aos de Alarcón et al $^{1}$ (3,6 dias), Forster et $\mathrm{al}^{8}$ (1,6 dia) e Orosz et $\mathrm{al}^{21}$ (19 horas). Em outros estudos, os tempos sugeridos para a realização da cirurgia foram de 24 a 48 horas, podendo chegar 
a 72 horas. ${ }^{11,12}$ Os motivos de uma espera maior para a operação podem ser em função da necessidade de se estabilizar clinicamente o paciente, no caso dos mais graves. ${ }^{21,25}$ Contudo, não foi possível identificar as razões dessa demora, se foi devido à gravidade do paciente ou a outras.

O efeito das comorbidades sobre a mortalidade pôde ser observado tanto em função de sua quantidade, quanto de sua gravidade, medida pelo ICC. Pacientes com mais doenças coexistentes tiveram maior chance de morrer, com um aumento dessa chance de $44 \%$ a cada nova patologia adquirida. A chance de óbito dos pacientes com escore ICC $>2$ foi cerca de seis vezes maior em relação ao escore zero. Outros estudos, como os de Cree et al, ${ }^{6}$ van Balen et al, ${ }^{24}$ Jain et al, ${ }^{15}$ e Grimes et al, ${ }^{12}$ também identificaram a influência das comorbidades sobre o óbito após a fratura proximal de fêmur. Segundo Cree et al, ${ }^{6}$ a taxa de mortalidade em 90 dias foi de $5 \%$ na presença de até três doenças associadas e $13 \%$ para quatro ou mais comorbidades. Em uma série histórica de 1992 a 1998, apresentada por Jain et al, ${ }^{15}$ as taxas de mortalidade em um ano após FPF de 1998 foram de $8 \%, 14,3 \%$ e $19,7 \%$ para escore de ICC 0,1 e 2 , respectivamente.

Clauge et al, ${ }^{4}$ ao estudarem a mortalidade hospitalar e em até 90 dias após a fratura proximal de fêmur, en- contraram associação entre o índice ASA e o aumento na chance de óbito. No presente trabalho, apesar de a taxa de mortalidade ter sido maior no grupo de pacientes classificados segundo o índice ASA como 3 ou 4, esse fator não representou um bom preditor do óbito. De todos os pacientes, $65,3 \%$ tiveram escore de até dois e apenas $2,0 \%$ foram classificados como ASA 4. Como esse o índice tenta avaliar a condição de saúde do indivíduo, esses achados podem indicar que a população estudada era menos debilitada, o que diminuiria o seu efeito. De modo semelhante, Ooi et $\mathrm{al}^{20}$ não associaram o índice ASA com a mortalidade ao avaliarem os resultados referentes a 84 pacientes, que em sua maioria tinham escore ASA 2, e o escore 4 foi o menos observado.

Concluindo, dos principais fatores citados pela literatura como preditores da mortalidade após FPF, o presente trabalho confirmou a idade e as comorbidades, além do tempo de espera entre a fratura e a cirurgia. Contudo, não foi possível distinguir se essa última variável é apenas um marcador de risco (pacientes mais graves têm a cirurgia postergada para compensação clínica) ou se a redução do tempo entre a fratura e a cirurgia poderia ter um efeito positivo na sobrevida dos pacientes. Entre as medidas de ajuste de risco, o índice ASA foi o único a não se apresentar como um bom preditor da mortalidade em 90 dias. 


\section{REFERÊNCIAS}

1. Alarcón T, González-Montalvo Jl, Bárcena A, Saez P. Further experience of nonagerians with hip fractures. Injury. 2001;32(7):555-8.

2. Casalleto JA, Gatt R. Post-operative mortality related to waiting time for hip fracture surgery. Injury. 2004;35(2):114-20.

3. Charlson ME, Pompei P, Ales KL, MacKenzie CR. A new method of classifying prognostic comorbidity in longitudinal studies: development and validation. J Chronic Dis. 1987;40(5):373-83.

4. Clauge JE, Craddock E, Andrew GA, Horan MA, Pendleton N. Predictors of outcome following hip fracture. Admission time predicts length of stay and in-hospital mortality. Injury. 2002;33(1):1-6.

5. Coeli CM, Camargo Jr KR. Avaliação de diferentes estratégias de blocagem no relacionamento probabilístico de registros. Rev Bras Epidemiol. 2002;5(2):185-96.

6. Cree M, Soskolne CL, Belseck E, Hornig J, McElhaney JE, Brant R, Suarez-Almazor M. Mortality and institutionalization following hip fracture. J Am Geriatr Soc. 2000;48(3):283-8.

7. D'Hoore W, Bouckeart A, Tilquin C. Practical considerations on the use of the Charlson Comorbidity Index with a administrative data bases. J Clin Epidemiol. 1996;49(12):1429-33.

8. Forster M, Calthorpe D. Mortality following surgery for proximal femoral fractures in centenarians. Injury. 2000;31(7):537-9.

9. Fransen $M$, Woodward M; Norton R, Robinson E, Butler M, Campbell J. Excess mortality or institutionalization after hip fracture: men are at greater risk then women. J Am Geriatr Soc. 2002;50(4):685-90.

10. Frohener M, Koch R, Litz R, Heller A, Oehlschlaeger $S$, Manfred, P. Comparison of the American Society of Anesthesiologists physical status classification with the Charlson score as predictors of survival after radical prostatectomy. Urology 2003; 62(4):698-701.

11. Gdalevich M, Cohen D, Yosef D, Tauber C. Morbidity and mortality after hip fracture: the impact of operative delay. Arch Orthop Trauma Surg. 2004;124(5):334-40.

12. Grimes JP; Gregory PM, Noveck H, Butler MS, Carson JL. The effects of time-to-surgery on mortality and morbidity in patients following hip fracture. Am J Med. 2002;112(9):702-9.

13. Hannan EL, Magaziner J, Wang JJ, Eastwood EA, Silberzweig SB, Gilbert M. Mortality and locomotion
6 months after hospitalization for hip fracture: risk factors and risk-adjusted hospital outcomes. JAMA. 2001;285(21):2736-42.

14. Iezzoni LI, Shwartz M, Ash AS, Hughes JS, Daley J, Mackiernan, YD. Severity measurment methods and judging hospital death rates for pneumonia. Med Care. 1996;34(1):11-28.

15. Jain R, Basisnski A, Kreder H. Nonoperative treatment of hip fractures. Int Orthop. 2003;27(1):11-7.

16. Lyons AR Clinical outcomes and treatment of hip fractures. Am J Med. 1996; 103 Suppl 1:S51-S64.

17. Nather GA, Seow CS, lau P, Chan A. Morbidity and mortality for elderly patients with fractured neck of femur treated by hemiarthrosplaty. Injury. 1995;26(3):187-90.

18. Nurmi L, Narinen A, Lüthje L, Tanninen S. Functional outcome and survival after hip fracture in elderly: a prospective study of 106 consecutive patients. I Orthopaed Traumatol. 2004;5(1):7-14.

19. O'hara DA, Duff A, Berlin JA, Poses RM, Lawrence VA, Huber EC et al. The effect of anesthetic technique on postoperative outcomes in hip fracture repair. Anesthesiology. 2000;92(4):947-57.

20. Ooi LH, Wong TH, Toh CL, Wong HP. Hip fractures in nonagenarians - a study on operative and nonoperative management. Injury. 2005;36(1):142-7.

21. Orosz GM, Magaziner J, Hannan EL, Morrison RS, Koval K, Gilbert M, et al. Association of timing of surgery for hip fracture and patient outcomes. JAMA. 2004;291(14):1738-1743.

22. Sakaki MH, Oliveira AR, Coelho FF, Leme LEG, Suzuki I, Amatuzzi MM. Estudo da mortalidade na fratura do fêmur proximal em idosos. Acta Ortop Bras. 2004;12(4):242-9.

23. Stoddart J, Horne G, Devane P. Influence of preoperative medical status and delay to surgery on death following a hip fracture. ANZ I Surg. 2002;72(6):405-7.

24. Van Balen R, Steyerberg EW, Polder JJ, Ribbers TL, Habbema JDF, Cools HJM. Hip fracture in elderly patients: outcomes for function, quality of life, and type of residence. Clin Orthop Relat Res. 2001;(390):232-43.

25. Zuckerman JD. Hip fracture. N Engl J Med. 1996;334(23):1519-25.

Artigo baseado na dissertação de mestrado de RC Souza, apresentada à Universidade Federal do Rio de Janeiro, em 2005. Financiado pelo Conselho Nacional de Desenvolvimento Científico e Tecnológico (CNPq - Processos 464042/2000-3 e 474845/2001-0). 